\title{
Early VLBI in the USSR
}

\section{Matveyenko L.I. ${ }^{1}$}

Space Research Institute

117997, Profsojuznaja 84/32, Moscow, Russia

E-mail: Imatveyenko@gmail.com

Abstract

This article gives the story of interferometer with independent elements in the USSR. At the end of February 1962 the author discussed with G.Ya. Gus'kov and E.G. Mirzabekjan, DSN Station, Evpatoria the possibility of a new type of radio interferometer, and proposed its realization between two DSN stations EVP-SMF. In September 1962 he reported the new method at a seminar of Radio Astronomical Laboratory, Pushino, and then together with N.Kardashev and G.Sholomitskii at a seminar of Astronomical Institute GAISH, which recommended taking out a Patent. In summer 1963 the author discussed with B. Lovell in DSN Evpatoria the VLBI method and signed memorandum an EVP-JB experiment at $\lambda=32 \mathrm{~cm}$. In December 1963 the Patent Bureau permitted publication, and the paper was sent to Radiofizika. The proposal of $M$. Cohen and K. Kellermann to do an experiment between 22-m antenna Pushino and 43-m Green Bank at $\lambda=3 \mathrm{~cm}$ accelerated the progress of VLBI in the USSR.

\section{Introduction}

Progress of technologies in the middle of the last century gave us high sensitivity radio telescopes - the foundation of modern radio astronomy, and fundamental discoveries of synchrotron and maser emission, recombination and molecular radio lines, quasars and pulsars. This was time of the beginning of the space exploration era, with direct studies of planets. An instrument with high angular resolution was needed for both astronavigation and studies of astronomical objects. But length of radio waves limited an angular resolution. The proposed method of "independent" interferometer - VLBI removed the problems and opened a new page of astronomy and applied directions. At the end of the 1950s the first space vehicles, Lunniks, were launched. Radio astronomers of the Lebedev Physical Institute (FIAN) measured trajectories of the space probes with radio interferometer [1]. The results of measurements were highly praised by the President of the Academy of Sciences of the USSR, M.V.Keldish and the Chief rocket designer S.P. Korolev. This technique enabled measurement of trajectories of the solar plasma flows [2]. During this time the construction of the deep-space tracking station was started near Evpatoria, in the Crimea. The FIAN collaborated in the creation of the interferometer formed by two ADU-1000 antennas, composed of eight 16-m parabolic dishes, mounted on a naval gun turret. Fig.1.

Resolving the Sky - Radio Interferometry: Past, Present and FutureManchester, UK April 17-20, 2012

1 Speaker 
The antennas were equipped with low-noise parametric preamplifiers at $\lambda=32 \mathrm{~cm}$ and masers at $\lambda=$ $8 \mathrm{~cm}$. The DSN had atomic frequency standards (ammonia type) and a magnetic tape recorder, with a bandwidth of $100 \mathrm{kHz}$. The angular accuracy of 0.1 " was achieved, a very high accuracy for that time. Compact radio sources were needed for calibration. The candidates were sources of relativistic particles, like the Crab Nebula that ejected relativistic jets [2]. These sources were suspected to be variable in radio emission. We invited G. S. Khromov and G. Sholomitskii to investigate this problem.

\subsection{High angular resolution}

The method of lunar occultation was at first a possibility. The occultation of the Crab Nebula took place on 16 April 1964. Our observations of the Crab Nebula transits across the ADU-1000 beam at $\lambda$ $=8 \mathrm{~cm}(15$ April) detected an extra feature in the pattern (an arrow in Fig. 1). This feature corresponds to a bright region located 1.5 light-years SE of the binary star. The nature of the variability of radio emission was explained by high directivity. The diffraction pattern at $32 \mathrm{~cm}$ was discovered, which corresponds to the star - the pulsar [3] (Fig. 1). Observations of an occultation of the 3C 273 at $\lambda=8$ $\mathrm{cm}$ and $32 \mathrm{~cm}$ determined the position and spectrum of the core and jet [4].
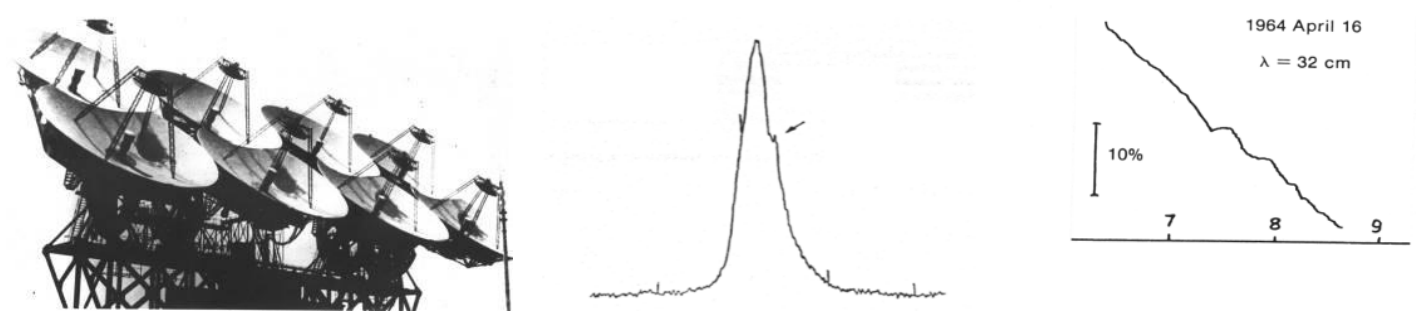

Fig.1. Antenna ADU-1000, DSN, Evpatoria. Transit of the Crab Nebula $\lambda=8 \mathrm{~cm}$, and diffraction pattern at $\lambda=$ $32.5 \mathrm{~cm}$, April 16, 1964.

\subsection{Interferometer with independent elements}

The occultation method has limited possibilities. Fifty years ago (at the end of February1962) I discussed with G.Ja.Gus'kov (head of DSN stations) and E.G. Mirzabekjan (Director of the Electronics Institute) long baseline Doppler measurements. It was decided to decrease frequencies and record data on a tape recorder, at that time the differential Doppler frequency was determined from computation. I proposed to add pilot signals of atomic frequency standards and determine the differential phase. In this case the elements of the interferometer are physically disconnected and distance between antennas is not limited. Gus'kov proposed to use DSN stations in Evpatoria and Simferopol at $32 \mathrm{~cm}$. In autumn 1962 I gave a talk titled "The Interferometer with Independent Elements" at the seminar of the Radio Astronomy Laboratory FIAN. My proposal for the experiment was not supported. After a few days I met with my colleagues from GAISh, G.Sholomitskii and N.Kardashev, who supported my idea, and gave a seminar in GAISh. The decision of the GAISh seminar was: "The idea is very important and should be patented". In December 1962 the proposal for the patent: L.I. Matveenko, N.S. Kardashev, and G.B. Sholomitskii: "Radio interferometer with long baseline" was sent to the Patent Bureau.

In summer 1963 I was received by Prof. B. Lovell who was a guest of M.V. Keldysh at DSN. We invited I.S. Shklovskii to support the astrophysical part and G.S. Khromov for translation [2]. I acquainted Prof. B. Lovell with the technical part, including transmitter, atomic standards, low-noise receivers, and radio astronomical studies. I.S. Shklovskii proposed that I talk about the VLBI method. B. Lovell agreed with me, but doubted the necessity of the very high resolution, because compact sources were unknown. We prepared Memorandum concerning interferometer Evpatoria - Jodrell Bank at $\lambda=32 \mathrm{~cm}$. Lovell proposed to discuss details at Jodrell Bank and to inform me of the outcome, but I did not receive any answer [5]. In December 1963 the Patent Bureau agreed with the 
publication and the paper which was submitted to the Radiofizika (Radiophysics) journal. The paper was accepted for publication after deleting one word: "satellite".

\subsection{Proposal of M.H. Cohen and K.I.Kellermann}

The VLBI was carried out in 1967 by two independent teams in the USA [7] and in Canada [8]. In February 23, 1968 M.H.Cohen and K.I. Kellermann sent a letter to V.V. Vitkevich (FIAN) with the proposal of VLBI at $\lambda=3 \mathrm{~cm}$ between Puschino and Green Bank Fig. 2. Vitkevich gave it to me and said: "This is your idea and you should be involved, but the chance is very small - the cold war time". Only through the support of N.G. Basov (Deputy Director of FIAN) did an experiment become possible. We proposed to use the 22-m antenna in Simeiz. A.B. Severnii, Director of the CRAO, supported us and we informed NRAO about this opportunity. I think it was D.Heechen who "opened" the green light. In January 1969 I.G. Moiseev and I visited NRAO to discuss technical requirements and the program of observations. For guarantee we set observations at $\lambda=6 \mathrm{~cm}$ and at $\lambda=3 \mathrm{~cm}$. Realization of VLBI required organization of a special division. FIAN could not achieve this. In June 1969 Director of Institute for Space Research (IKI) G.I. Petrov met with Director of FIAN D.V. Skobel'tsyn, and N.G. Basov, and they decided to transfer VLBI to IKI, where the first VLBI laboratory (Matveyenko) was organized.
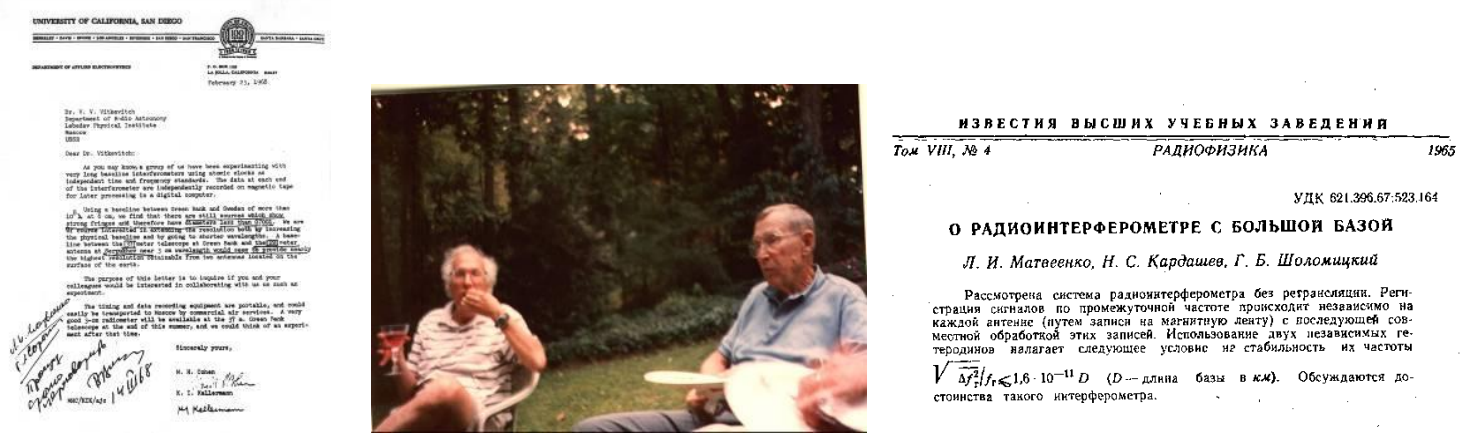

Fig. 2. Letter of M.H.Cohen and K.I. Kellermann, 1967 with Vitkevich's remark - left. K.I.Kellermann and D.Heechen. The paper was submitted in 1963 and published in 1965 [6].

\subsubsection{CR-GB observations}

In autumn 1969 K.I. Kellermann, B.Clark and J.Payne visited Crimea Fig. 3. Rubidium clock was transported to Simeiz for time synchronization. Near Yalta police stopped the car as we were speeding. The policeman asked to show the atomic clock. The clock had stopped! K. Kellermann asked Olof Rydbeck to send working rubidium standard. After a few days interferometer fringes at 6 $\mathrm{cm}$ were detected $[2,9]$.
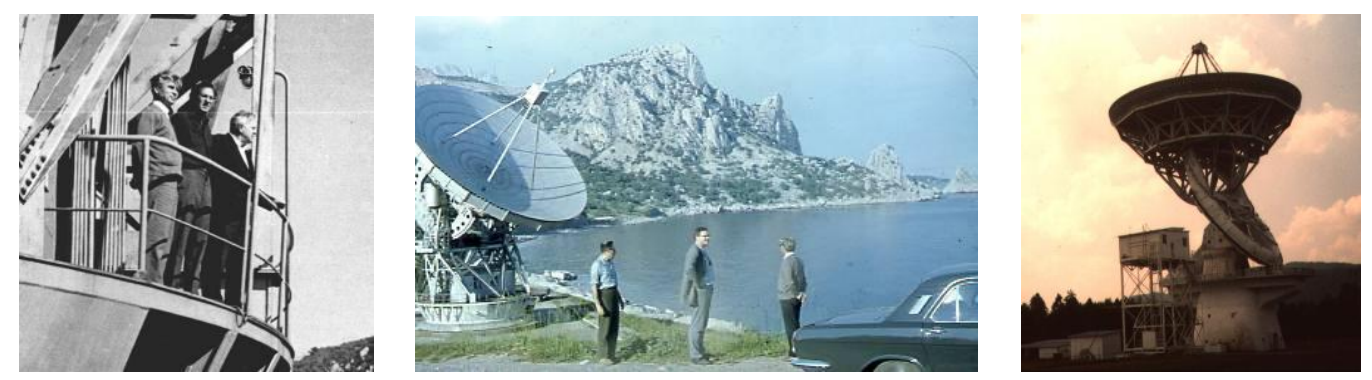

Fig. 3. RT-22 CR: I.G.Moiseev, K.I.Kellermann, and V.V.Vitkevitch - left. M.H.Cohan, B.Clark, and I.G.Moiseev, and RT-43, GB. 
Data processing was done at NRAO IBM 360/60. The $2.8 \mathrm{~cm}$ data was independently correlated on fast computers IBM 360/75 in Caltech and in GSFC. Fringes were seen from 3C 273 and 4C 39.25 [9]. The observations showed that intercontinental VLBI was possible at centimeter wavelengths, and quasars had complex structure. They required observations with a range of baseline lengths, and high sensitivity.

\subsubsection{CR-GB-GST observations at $3.5 \mathrm{~cm}$}

The next experiment was in June 1971 at $\lambda=3.5 \mathrm{~cm}$ and included the 64-m DSN antenna in Goldstone (Fig. 6). Participants of the experiment at RT-22 Simeiz, M.Cohan, B.Clark and Moiseev are seen in Fig. 5. Cassegrain system of an antenna and a low-noise maser preamplifier were made at RT-22 (Fig. 6). NRAO produced a correlator and a broadband registration system MK-2. To improve frequency stability $\mathrm{M}$. Cohen combined a Rubidium standard with a special crystal oscillator. The fringes of 3C 273 at baseline CR-GST are shown in Fig. 6. We discovered superluminal velocities of components and brightness temperature $\mathrm{T}_{\mathrm{b}}=10^{12} \mathrm{~K}$, that corresponded to the inverse Compton scattering [11]. The VLBI opened a new epoch for studies of fine structure of astronomical objects and solving many applied problems at a principally new basis. NRAO began building VLBA.
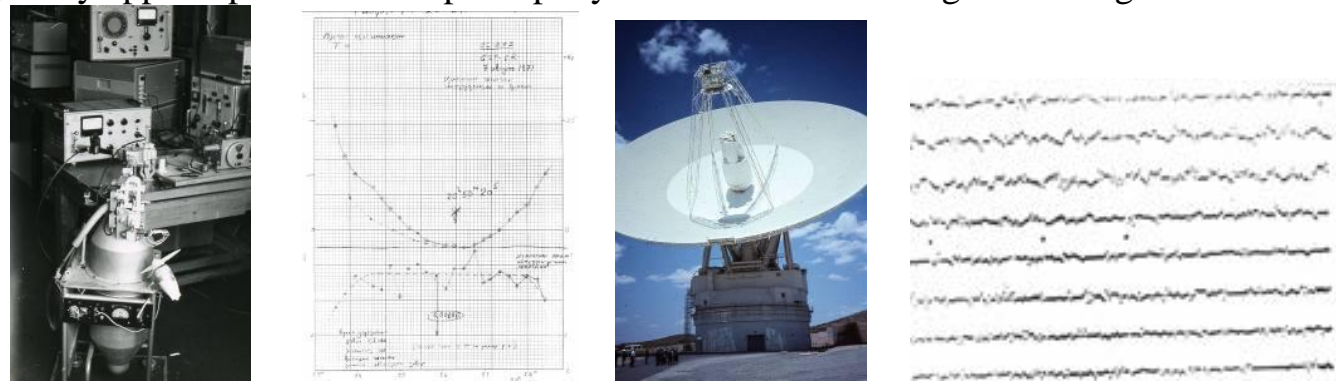

Fig. 6. Maser preamplifier $3.55 \mathrm{~cm}$ - left. Next - discovering fringes of 3C 273 at $3.55 \mathrm{~cm}$ CR-GST. Antenna GST. Fringes of 3C 273.

\subsection{The AGN objects}

The galaxy nuclei are surrounded by thermal plasma, visible in an optical emission lines. The fine structure of $3 \mathrm{C} 345$ at $\lambda=1.35$ to $\lambda=92 \mathrm{~cm}$ showed that this medium absorbed synchrotron emission and determined the cutoff frequencies in spectra of the core and nearest components (Fig. 7). The cut off frequency decreased with the angular distance from the core [14]. Nearest compact part of the core is visible at millimeter wavelengths and the large scale structure of jet can be seen at low frequencies. The scattering in the interstellar medium has limited an angular resolution [18].
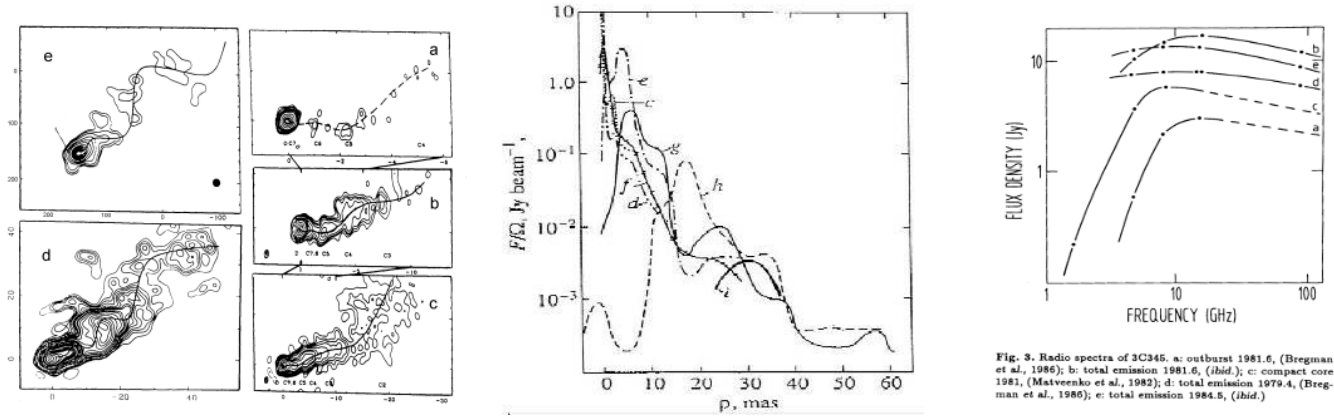

Fig. 7. QSS 3C 345, maps at wavelengths $1.35-92 \mathrm{~cm}$, strip brightness distribution, and spectra of compact components [14]. 
The thermal plasma was detected from absorption recombination radio lines [17]. The surrounded thermal plasma determines the rotation measure [14]. The brightness temperature of the compact component reaches $10^{12} \mathrm{~K}$. Superluminal velocities of components and their accelerations were observed. Instability of ejected outflow determined the helix structure of the jet.

In 1993 observations of AGN object $1803+784$ were conducted at $18 \mathrm{~cm}$ with a 20-stations global VLBI network, which included the 70-m, 64-m and two 22-m antennas. Maps with high angular resolution are shown in Fig. 8 [13]. The jet has a helix structure with variable step determining instability of the outflow (Fig 8 right).
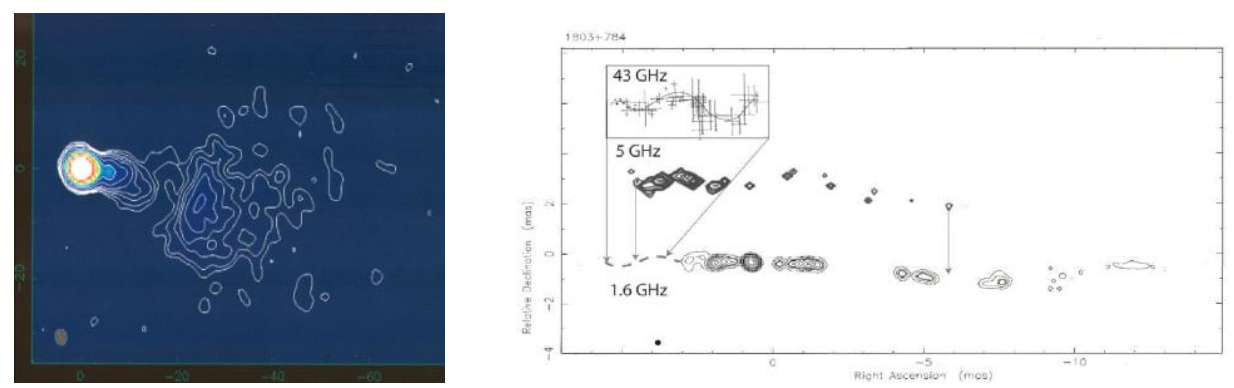

Fig. 8. AGN object $1803+784, \lambda=18 \mathrm{~cm}$, global VLBI, $\varphi=0.1$ mas - left, and $\varphi=0.05$ mas - right [13].

\subsection{The $\mathrm{H}_{2} \mathrm{O}$ objects}

In February 1969 at a seminar in Berkeley, Jack Welch announced the discovery of the water vapor emission at $\lambda=1.35 \mathrm{~cm}$. It was explained in terms of the thermal emission, but for us these sources had been the best candidates for VLBI measurements: narrow line width and very high flux densities. I discussed with J.Welch the possibility of VLBI measurements on the USSR-USA interferometer for study emission mechanism. We continued this discussion at MIT with B.F. Burke, J. Moran, and engineer Kosmo-Papa on details of experiment CR-HST. The main problem was local oscillator stability. We decided to begin testing equipment on the short baseline within the USA, and then move to intercontinental baselines [2].

\subsubsection{Observations CR-HST}

In June 1971 we observed $\mathrm{H}_{2} \mathrm{O}$ objects with angular resolution 0.1mas. At this time a powerful outburst occured in the object W49. Rapidly increasing flux density corresponded to a compact region. The $\mathrm{H}_{2} \mathrm{O}$ line profile received at the RT-22 CR is shown in Fig. 9. The outburst region was resolved partially, and the brightness temperature was determined to be as high as $\mathrm{T}_{\mathrm{b}}=10^{16} \mathrm{~K},[16]$.
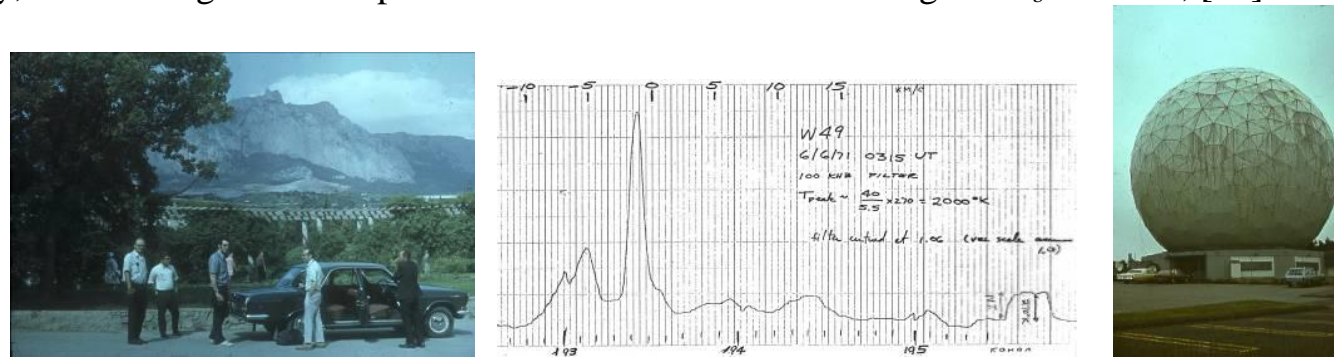

Fig. 9. B. Burke (MIT), S. Knowles (NRL), J. Moran (SAO), and Cosmo-Papa (MIT) at Miskhor (USSR). Profiles of W49 line emission, and antenna Haystack. 


\subsubsection{Space VLBI, $\lambda=1.35 \mathrm{~cm}$}

The nature of the super radiation from the $\mathrm{H}_{2} \mathrm{O}$ maser outburst in W 49 had no explanation. The extra high angular resolution was needed. Director of IKI, G.I.Petrov 9.02.1972 support to design a spaceground interferometer at $\lambda=1.35 \mathrm{~cm}$. The director of the project was V.P. Mishin (Head of Lavochkin's company), scientific head, L.I.Matveenko and designer, Valery I. Kostenko. A diameter of antenna, 3.1-m, was determined by the rocket size.
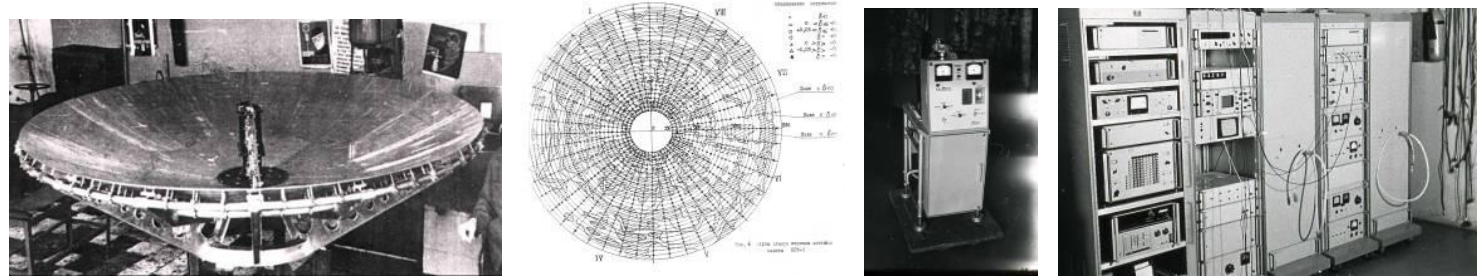

Fig.10. Parabolic antenna of SRT-3.2 $\mathrm{m}$ and map of the surface accuracy. Maser preamplifier at $1.35 \mathrm{~cm}$ and hydrogen frequency standard.

The reflecting surface was reinforced with a thermo-isolated framework and with thin invar pivots. That kept its shape despite large changes of temperature (Fig. 10). The accuracy of the surface was good enough for the sub-mm band. The secondary mirror could compensate a change of the focal position and the fine pointing of the antenna [17]. The apogee of $20,000 \mathrm{~km}$ was determined by the high-energy particles and non-uniform gravity field of the Earth, the perigee of 70,000 km by the space radio telescope sensitivity.
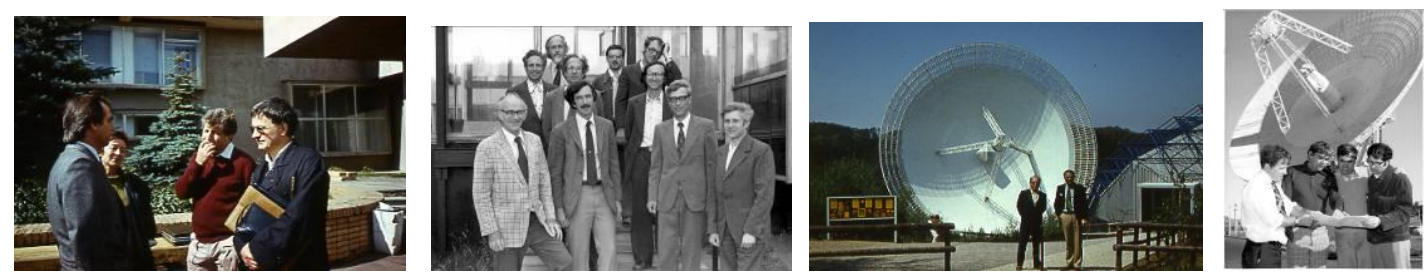

Fig. 11. Peter Mezger and Rolf Schwartz; the VLBI team, IKI; I. Shklovskii and R. Wielebinskii at RT-100-m, Effelsberg and 64-m antenna DSN, Tidbinbilla: S.Knowles (NRL), D. Jauncey, K. Wellington, and V. Kostenko.

The project had a broad international cooperation, which included the USA, Australia, India, and European countries $[2,28]$. One of a Soviet-FRG work meeting had been in Moscow. P.Mezger was head of the FRG delegation and me of the USSR group (Fig. 11). The USA partners had been too, namely, B.Burke and Bob Preston. The limiting of baseline by scattering of interstellar medium and sensitivity was discussed [18]. N.Kardashev and V.Slish talked about space VLBI at $18 \mathrm{~cm}$.
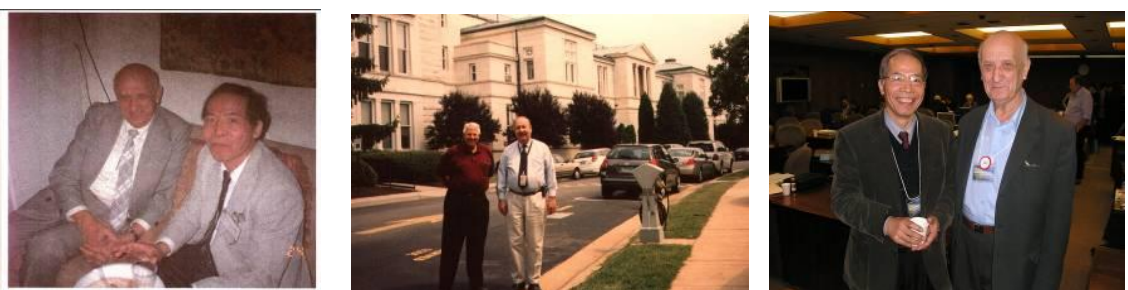

Fig. 12. Discussing the space-ground experiment: E. Hirabajashi and L. Matveenko, Socorro; W.Howard III and K.Jonston, Naval observatory. The discussion of the VSOP results and future $\mathrm{H}_{2} \mathrm{O}$ observations, Tokyo.

The same time we worked for a ground VLBI network. For increased sensitivity radio telescopes Simeiz and Pushino were equipped with maser preamplifiers, and hydrogen frequency standards (Fig. 10). We regretted that we could not send the maser to Effelsberg and later equipped 70-m in Evpatoria with the maser. The RT 70 at $1.35 \mathrm{~cm}$ had $\mathrm{A}_{\text {eff }}=1500 \mathrm{~m}^{2}$ and $T_{\text {sys }}=60 \mathrm{~K}$. 
We had been continuing study of $\mathrm{H}_{2} \mathrm{O}$ objects [15, 19 - 22]. A 10-m radio telescope, in Bangalore was used to test the space-ground VLBI. The VLBI data was processed in MPIFR.

Our conclusions had some influence on the Japanese VLBI Space Observatory Programme (VSOP). The VSOP spacecraft was launched in 1997, and confirmed the optimal technological and operational parameters of an Earth-Space interferometer. We met with H.Hirobajashi, Head of the VSOP Project, in Socorro before launch and after launch in Tokyo to discuss results of observations (Fig.12).

\subsubsection{Orion KL}

The $\mathrm{H}_{2} \mathrm{O}$ maser emission accompanies the star formation. The super maser outbursts in Orion KL had been in 1979-1987, radiation reached 8 MJy! The outbursts emission was determined by a chain of compact components, distributed along S-form structure (Fig. 13). The structure corresponds to a disk seen edge-on, with the rotation period of $\mathrm{T}=170 \mathrm{yr}$. The surrounding matter accreting to the disk moved along arms and ejected to bipolar outflow. Tangential directions of the arms correspond to compact sources, brightness temperature of which are $\mathrm{T}_{\mathrm{b}} \approx 10^{12} \mathrm{~K}$. The extra high temperature is result of amplification more than two orders of magnitude in the neighborhood [15, 21]. Extra ordinary rotation of polarization is determined by different orientation polarization at nozzle outputs and velocities of outflows [23].
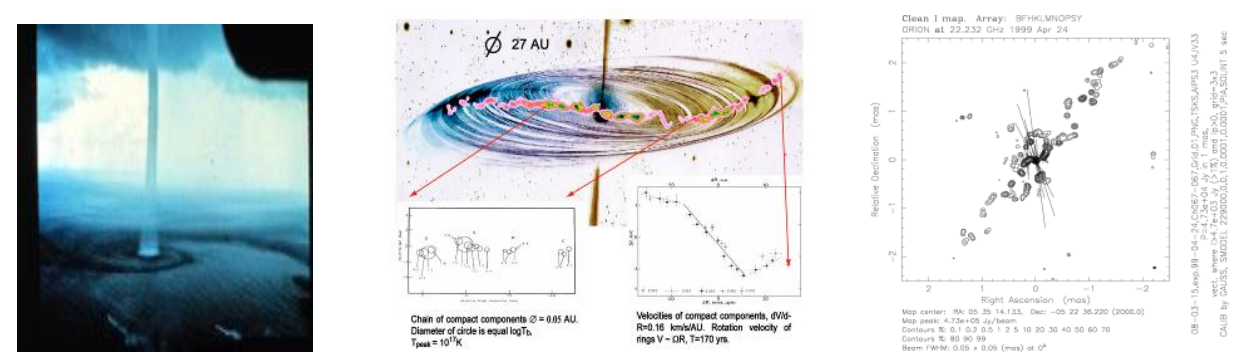

Fig. 13. Structure of star formation region in Orion KL

\subsection{VEGA}

In 1974 we proposed VLBI methods to study the dynamics of the Venus atmosphere - the VEGA project [24 - 26]. The USSR network included antennas: 70-m Ussuriysk, and Evpatoria; 25-m Ulan Ude; 22-m Simeiz and Pushino; 64-m Bear Lakes. Radio telescopes had hydrogen frequency standards and MK-2 terminals. They were worked with global VLBI network at $\lambda=18 \mathrm{~cm}$. The interferometers were tested with W3 OH (analog of balloon transmitter) and QSS 3C 345, (Fig. 14).
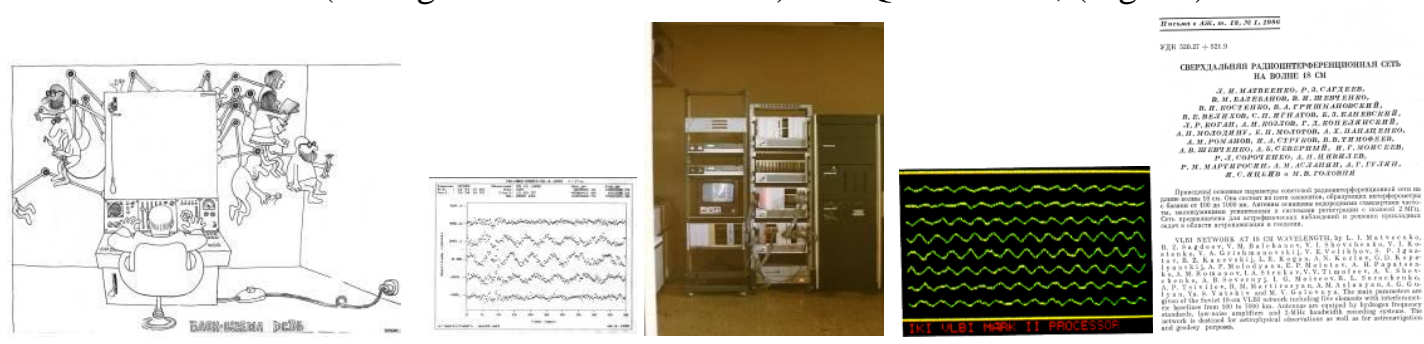

Fig. 14. The IKI VLBI team is working. A correlator and fringes of $\mathrm{W} 3 \mathrm{OH}$ and $3 \mathrm{C} 345$ at USS-EVP, [25].

The balloons were injected into the Venus atmosphere on June 11th and 15th 1985 (Fig. 15). The balloons drifted at an altitude of $53 \mathrm{~km}$ parallel to the Venus equator, with velocities of 69 and $67 \mathrm{~m} / \mathrm{s}$. Near Mount Aphrodite convection currents moved VEGA-2 balloon by about $1.1 \mathrm{~km} \mathrm{NW} \mathrm{[26].}$ 

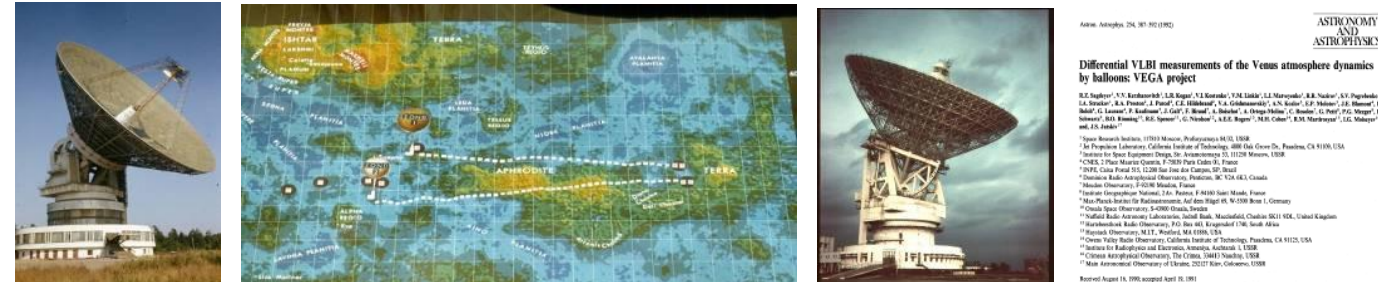

Fig. 15. Radio telescopes Bear Lake and Evpatoria. Trajectories of ballons in Venus [26].

We are continuing work for building 3 extra 70-m antennas, including India and Sufa; it was designed the 30-m space antenna of umbrella type [27], however the "Perestroika" was coming...

Acknowledgements. VLBI is the result of cooperation of people from different countries and continents. The Soviet VLBI is a part of this complex work. I am grateful to all my colleagues who accompanied and assisted us. I have not been able to personally thank all those involved, but would like to express my gratitude to Richard Schilizzi, who was a key figure in the success of the global VLBI Network. I am grateful to Sergei Gulyaev for comments on the paper.

\section{References}

[1] Vitkevich, V.V., Kuzmin A.D., Matveenko L.I., et al. 1961, Radiotechnika and Electronics. 9, p.1421.

[2] Matveenko L.I. Astron. Nachr. / AN 328, No. 5, 411 - 419 (2007) / DOI 0.1002/asna.20071076.

[3] Matveenko, L.I. 1975, PAZh, 7, p.13.

[4] Sholomitskii, G.B., Sleptsova, N.F., Matveenko, L.I.: 1965, Sov.Astr.J. 6, 1135.

[5] Bernard Lovell. Out of the zenith Jodrell Bank, Oxford Univ. Press 1973, p. 47.

[6] Matveenko, L.I., Kardashev, N.S., Sholomitskii, G.B.: 1965, Radiofizika 4, 651

[7] Clark B.G., Kellermann K.I., Cohen M.H., Jauncey D.L. 1967, Science. 157,189.

[8] Broten N.W., Legg T.H., Locke J.I., et.al. 1967, Science, 156. 48.

[9] Broderick, J.J., Vitkevich, V.V., Jauncey D.L., et al., 1970, Sov.Astr.J. 4, p. 784.

[10] Clark, B.F., Broderick, J.J., Efanov V.A., et al. 1972. 4, 700.

[11] Kellermann, K.I., Pauliny-Toth, I.I.K.: 1969, ApJ. 155, L31.

[12] Matveenko, L.I., Pauliny-Toth I.I.K., Bath L.B. et al. 1996, A\&A 312, 738.

[13] Britzen,S., Witzel, A., Krichbaum T.P., et al., 2001. Sov.Astron.Let. 1. 1.

[14] Matveenko,L.I.,Witzel, A. 1999, Sv.AJ, 9. p. 643.

[15] Matveenko, L.I., Zaharin k.M., Diamond P.J., Graham D.A. 2004. Sov. Astron.Let. 2, p. 100.

[16] B.F.Burke, K.J.Johnston, V.A.Efanov et al., 1972. Sov.Astronomy J., 3, 379.

[17] Kostenko,V.I., Matveenko,L.I., 1982. Cosmich. Studies, 20, 149 ; 1977. Preprint IKI, N 340.

[18] Matveyenko Preprint IKI, № 479, 1978, 1-28.

[19] Genzel R., Downes D.J. , Moran J.M. et al. 1978, A\&A, 66, 13.

[20] Genzel R., Downes, D.J., et al.: 1979, A\&A 233, 79

[21] Matveenko, L.I., Diamond, Ph,D., Graham, D.A., 1998, Astron. Lett., 10, 723.

[22] Demichev,V.A., Zaharin,K.M., Matveenko, L.I., 2003, Astron. Report, 80, 2, 118.

[23] Matveenko, L.I., Sivakon'S.S. 2006, Astronomy Report, 12, 951.

[24] Kogan, L.R., Matveenko, L.I., et al.: 1974, Symposium EOS-VENUS, Moscow, p. 1.

[25] Matveenko, L.I., Sagdeev,R.Z., Balebanov,V.M., et al.: 1986, PAZh 12, 59

[26] Sagdeev, R.Z., Kerzhanovitch V.V., Kogan L.R., et. al., 1992, A\&A. 254, 387.

[27] Savin, A.I., Zaxon, M.B., Matveenko, L.I.: 1991, in: T.J. Cornwell, R.A. Perley (eds.), Radio Interferometry: Theory.

[28] Burke B. 2007. Early days of VLBI in Space Astronomical Society of the Pacific Conference series.

Vol 402. p.10 eds. Y. Hagivara, E.Fomalont, M.Tsuboi, and Y.Murata. 